\title{
Transient atelectasis due to hilar lymph node swelling affected by lenalidomide-induced tumor flare reaction
}

\author{
Takahiro Suyama, ${ }^{1)}$ Terue Yui, ${ }^{1)}$ Atsuo Horiuchi, ${ }^{11}$ Rie Irie, ${ }^{2)}$ Yoshiyuki Osamura, ${ }^{2)}$ Naoki Miyao ${ }^{1)}$
}

Tumor flare reaction (TFR) is a unique immune-mediated tumor recognition phenomenon presenting as rapid enlargement of the tumor, which mimics disease progression, developing in the early stage of treatment using immunomodulatory drugs or immune checkpoint inhibitors. A 59-year-old man with follicular lymphoma had residual tumor burden in the left hilar lymph nodes after R-CHOP therapy, and received lenalidomide and rituximab $\left(\mathrm{R}^{2}\right)$ therapy. He developed respiratory distress on day 11 of $\mathrm{R}^{2}$ therapy. Chest X-ray and CT demonstrated left lung atelectasis due to left hilar lymph node swelling. We performed transbronchial lung biopsy on day 20 of $\mathrm{R}^{2}$ therapy. The biopsied left bronchus tissue exhibited extensive necrosis, which had a B-cell phenotype consistent with that of follicular lymphoma. Neither NK cells nor cytotoxic T cells were detected. It was unclear whether the immune effector cells disappeared at the time of transbronchial lung biopsy. Atelectasis in our patient improved by continuing $\mathrm{R}^{2}$ therapy beyond TFR.

Keywords: Tumor flare reaction, Pseudoprogression, Immunomodulatory drug

\section{INTRODUCTION}

Lenalidomide, an immunomodulatory drug, was reported to reactivate dysfunctional T and natural killer (NK) cells ex vivo by increasing their proliferative capacity and T-helper cell type 1 (Th1) cytokine release. ${ }^{1}$ Tumor flare reaction (TFR) is a unique immune-mediated tumor recognition phenomenon presenting as an increase in tumor burden, lowgrade fever and rash. Lenalidomide-induced TFR involves the activation of NK cells and T cells, and their infiltration into the tumor sites. ${ }^{2}$

TFR was originally described in patients with chronic lymphocytic leukemia (CLL) treated using immunomodulatory drugs (IMiDs) (thalidomide and lenalidomide) $)^{3,4}$ TFR was also observed in mantle cell lymphoma, indolent nonHodgkin lymphoma (NHL), aggressive NHL and Hodgkin lymphoma treated by lenalidomide. ${ }^{5}$

TFR mimics disease progression on imaging before an effective anti-tumor response occurs. A similar phenomenon, 'pseudo-progression', was also reported in multiple solid tumor types treated using immune checkpoint inhibitors (ICIs) resulting from T cells infiltrating the tumor site. ${ }^{6}$

We report a patient with refractory follicular lymphoma who exhibited transient atelectasis due to hilar lymph node swelling affected by lenalidomide-induced TFR.

\section{CASE REPORT}

A 58-year-old man visited a hospital in October 2019 for lymph node swelling and the left inguinal lymph node was biopsied. He was diagnosed with follicular lymphoma grade 3a (Figure $1 \mathrm{~A}-\mathrm{B}$ ) and referred to our hospital. We diagnosed his lymphoma as follicular lymphoma grade $3 \mathrm{a}$, stage IV and FLIPI: high. We administered bendamustine, but it was ineffective. He then received R-CHOP therapy and had a partial response. After 5 courses of R-CHOP therapy, left hilar lymph node swelling remained on PET/CT with a SUV of 20.5 as the main lesion (Figure 2).

We started $\mathrm{R}^{2}$ (lenalidomide at $20 \mathrm{mg} /$ day, days 1-21 and rituximab at $375 \mathrm{mg} / \mathrm{m}^{2}$, day 1) therapy in June 2020 (day 1). At that time, the WBC count was $3,870 / \mu 1(3,500-8,500)$, containing $2,593 / \mu \mathrm{l}$ of neutrophils and $368 / \mu$ of monocytes. $\mathrm{Hb}$ was $12.4 \mathrm{~g} / \mathrm{dl}(11.5-17.0)$, platelet count was $25.4 \times 10^{4} / \mu \mathrm{l}$ (15.0-35.0), LDH was $387 \mathrm{U} / \mathrm{L}(120-200)$ and CRP was 0.57 $\mathrm{mg} / \mathrm{dl}(\leq 0.30)$. He was in a good condition on day 4 of $\mathrm{R}^{2}$ therapy. Eleven days after starting $\mathrm{R}^{2}$ therapy, he visited our hospital for respiratory discomfort. His body temperature was $36.5^{\circ} \mathrm{C}$, blood pressure was $129 / 62 \mathrm{mmHg}$ and $\mathrm{SpO} 2$ (room air) was $89-90 \%$. He exhibited grade 1 rash, but had no pain. The WBC count was $2,120 / \mu 1$, containing $922 / \mu$ of neutrophils and $424 / \mu$ l of monocytes. The monocyte count

\footnotetext{
Received: September 16, 2020. Revised: November 10, 2020. Accepted: December 7, 2020. J-STAGE Advance Published: January 8, 2021 DOI: $10.3960 /$ jslrt.20045

${ }^{1}$ Department of Internal Medicine and ${ }^{2}$ Pathology, Nippon Koukan Hospital, Kawasaki, Kanagawa, Japan

Corresponding author: Takahiro Suyama, Department of Internal Medicine, Nippon Koukan Hospital, 2-1, Koukandouri, 1-chome, Kawasaki-ku, Kawasaki-si, Kanagawa 210-0852,

Japan. E-mail: takahiro-suyama@koukankai.or.jp

Copyright (C) 2021 The Japanese Society for Lymphoreticular Tissue Research

(cc) BY-NC-SA This work is licensed under a Creative Commons Attribution-NonCommercial-ShareAlike 4.0 International License.
} 
A

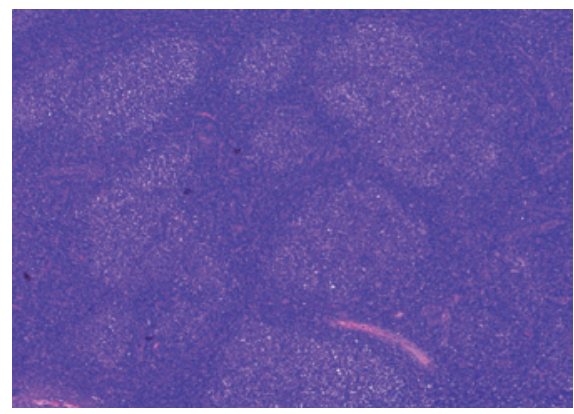

$\mathrm{D}$

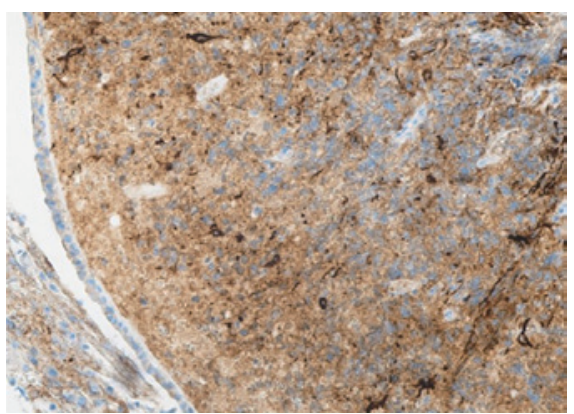

G

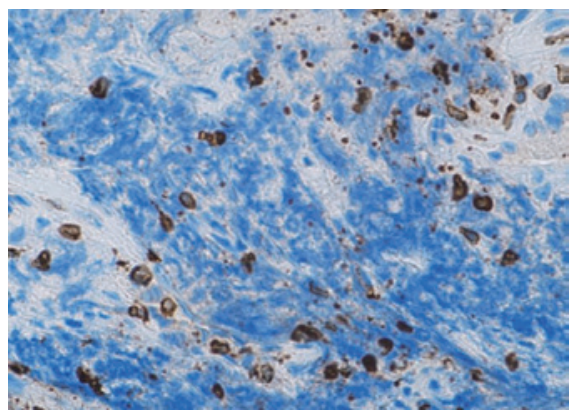

J

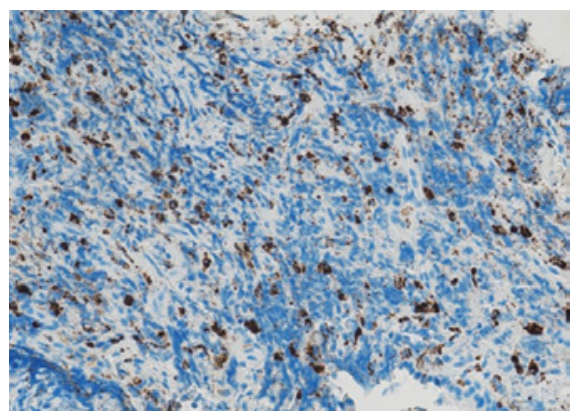

B

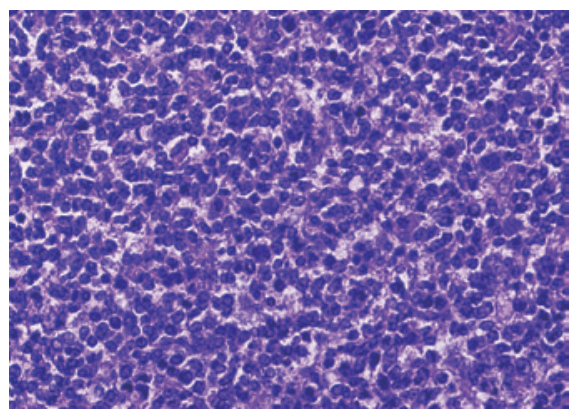

$\mathrm{E}$

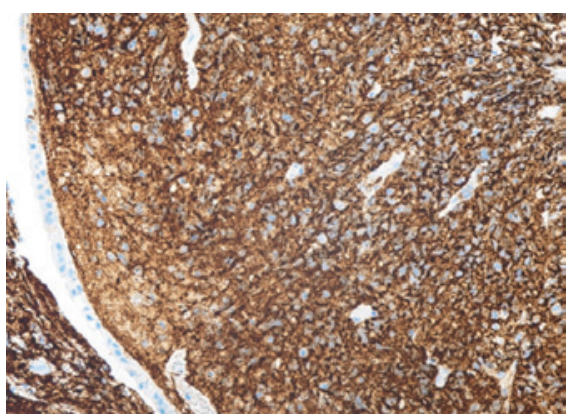

$\mathrm{H}$

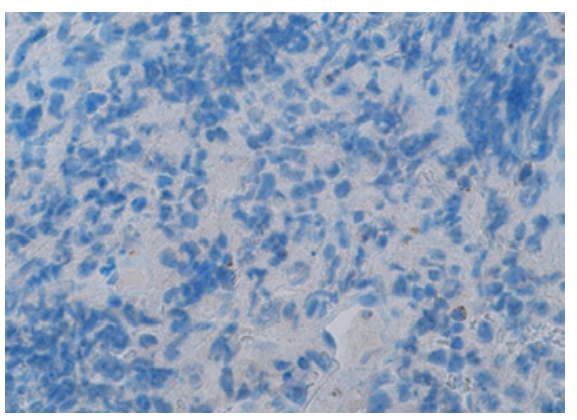

C

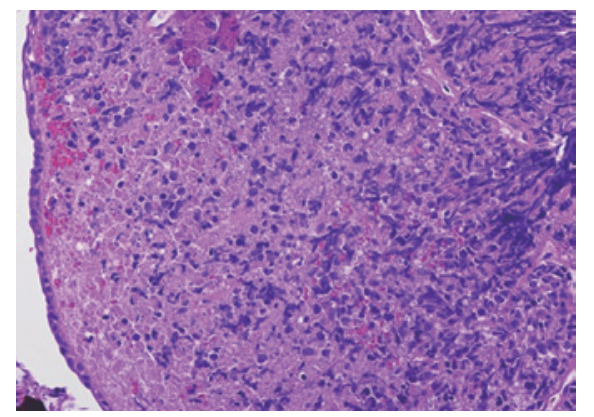

F

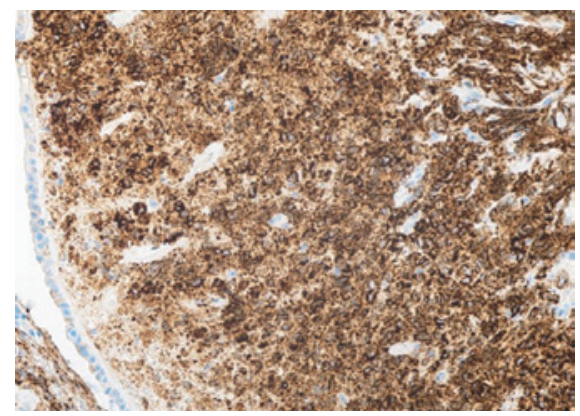

I

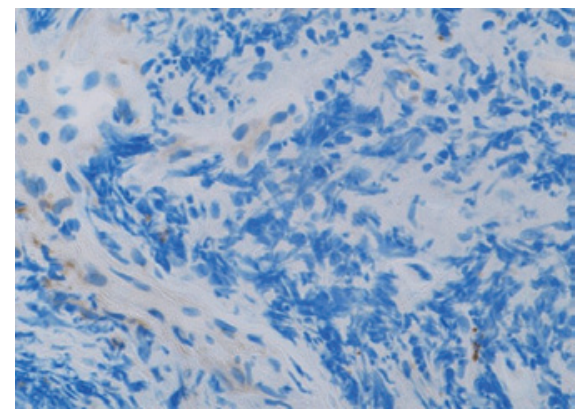

Fig. 1. Pathological findings: The inguinal lymph node at onset $(\boldsymbol{A} \times 40, \boldsymbol{B} \times 400)$; The neoplastic follicles show a vaguely nodular pattern $(\boldsymbol{A})$. Both centrocytes and centroblasts were present $(\boldsymbol{B})$.

The biopsied left bronchus tissue (C-J × 200); On hematoxylin and eosin staining, dense infiltration of lymphocytes, which were almost all necrotic, was observed under the bronchial epithelium $(\boldsymbol{C})$. Although they had necrotic change, they were CD10-positive $(\boldsymbol{D})$, CD20-positive $(\boldsymbol{E})$, PAX-5-positive (not shown) and bcl-2-posistive cells $(\boldsymbol{F})$, which is consistent with the phenotype of follicular lymphoma. There were few CD3-positive cells $(\boldsymbol{G})$, which were negative for granzyme B $(\boldsymbol{H})$, i.e., not cytotoxic T cells. CD56-positive cells, i.e. NK cells, were not detected (I). CD68-positive cells considered to be macrophages were well noted $(\boldsymbol{J})$. 


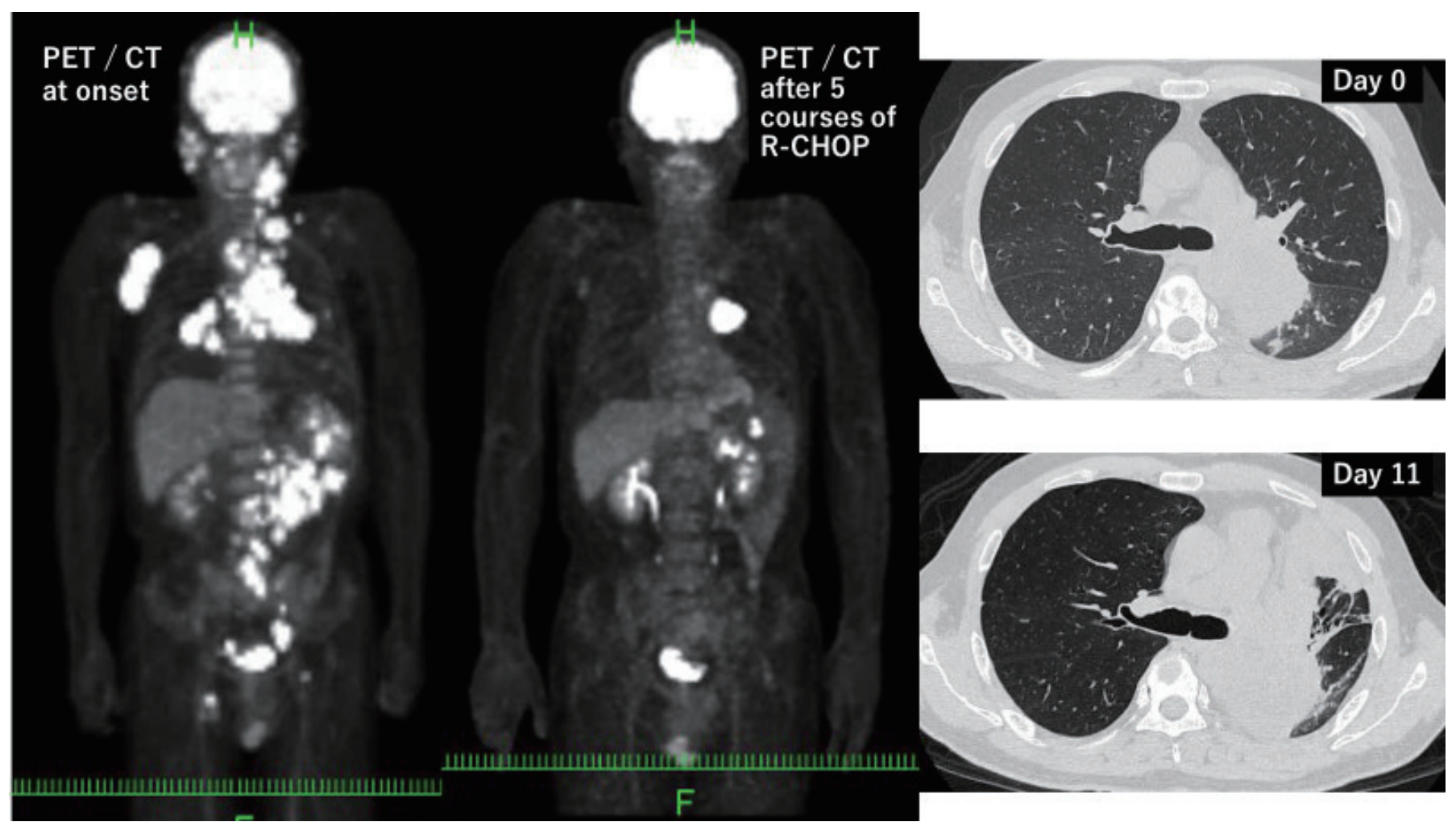

Fig. 2. Radiographic findings: PET/CT at onset showed abnormal uptake in the extensive lymph nodes (left). Residual abnormal uptake in the left hilar lymph node was noted after 5 courses of R-CHOP therapy (middle). CT on day 0 showed enlargement of the left hilar lymph node (upper right). Further enlargement, obstructive atelectasis of the left lung and mediastinal shift to the left were observed on CT on day 11 (lower right).

was relatively high at $20 \%$ of the WBC. Hb was $13.6 \mathrm{~g} / \mathrm{dl}$, platelet count was $29.3 \times 10^{4} / \mu \mathrm{l}$, LDH was $264 \mathrm{U} / \mathrm{L}$ and CRP was $0.99 \mathrm{mg} / \mathrm{dl}$. Chest X-ray revealed a left lung severe shadow (Figure 3). CT demonstrated left lung atelectasis due to left hilar obstruction by lymph node swelling (Figure 2).

After emergency hospitalization on day 11 of $\mathrm{R}^{2}$ treatment, he was stable on oxygen inhalation of $0.5 \mathrm{~L} / \mathrm{min}$ at rest and $2 \mathrm{~L} / \mathrm{min}$ when walking. Antihistamine was prescribed for rash. We continued the combination lenalidomide-rituximab $\left(\mathrm{R}^{2}\right)$ immunotherapy. Although rituximab was administered 6 times through 5 courses of R-CHOP therapy and on day 1 of this course, it was added on day 13. The left hilar lymph node swelling and atelectasis did not improve on chest $\mathrm{X}$-ray on day 18 (Figure 3). If the obstruction of the left bronchus was not due to TFR but to true progression, radiation therapy was considered necessary. We thus carried out transbronchial lung biopsy on day 20 of $\mathrm{R}^{2}$ treatment. The biopsied left bronchus tissue exhibited dense infiltration of lymphocytes, which were almost all necrotic. Although they had necrotic change, their phenotype was consistent with that of follicular lymphoma. Neither NK cells nor cytotoxic T cells were detected. Macrophages were well noted. (Figure $1 \mathrm{C}-\mathrm{J})$.

Rituximab was added just after transbronchial lung biopsy on day 20. From around this time, his respiratory state improved. Chest X-ray on day 25 revealed a decrease in left hilar lymph node swelling and improvement of atelectasis. He no longer needed oxygen inhalation and was discharged. As chest X-ray on day 28 (Figure 3) revealed left lung expansion, he received a second cycle of $\mathrm{R}^{2}$ therapy from day 29. The left lung atelectasis expanded through the second cycle of $\mathrm{R}^{2}$ therapy (Figure 3 ). Central nervous system involvement developed thereafter and we changed $\mathrm{R}^{2}$ therapy to R-CHASE therapy, which contains high-dose cytarabine, and intrathecal administration of methotrexate, cytarabine and prednisolone.

\section{DISCUSSION}

TFR is acutely dependent on NK cell function, and is then maintained by the rapid recruitment and proliferation of T cells. ${ }^{2}$ Andritsos et al. reported increased CD3-positive, CD4-positive, CD8-positive and granzyme B-positive T-cells in an excised swollen tonsil after lenalidomide treatment. In the present case, the biopsied left bronchus tissue on day 20 of $\mathrm{R}^{2}$ therapy did not contain NK cells or cytotoxic $\mathrm{T}$ cells. Macrophages were relatively conspicuous. It is unclear whether NK cells and cytotoxic T cells disappeared at the time of transbronchial lung biopsy when the respiratory state began improving. Although it is possible that the left hilar lymph nodes swelled due to tumor progression without TFR, rapid enlargement just after the start of $\mathrm{R}^{2}$ therapy may have been related to TFR.

$\mathrm{R}^{2}$ therapy was reported to have favorable activity in patients with relapsed/refractory follicular lymphoma. ${ }^{8}$ 


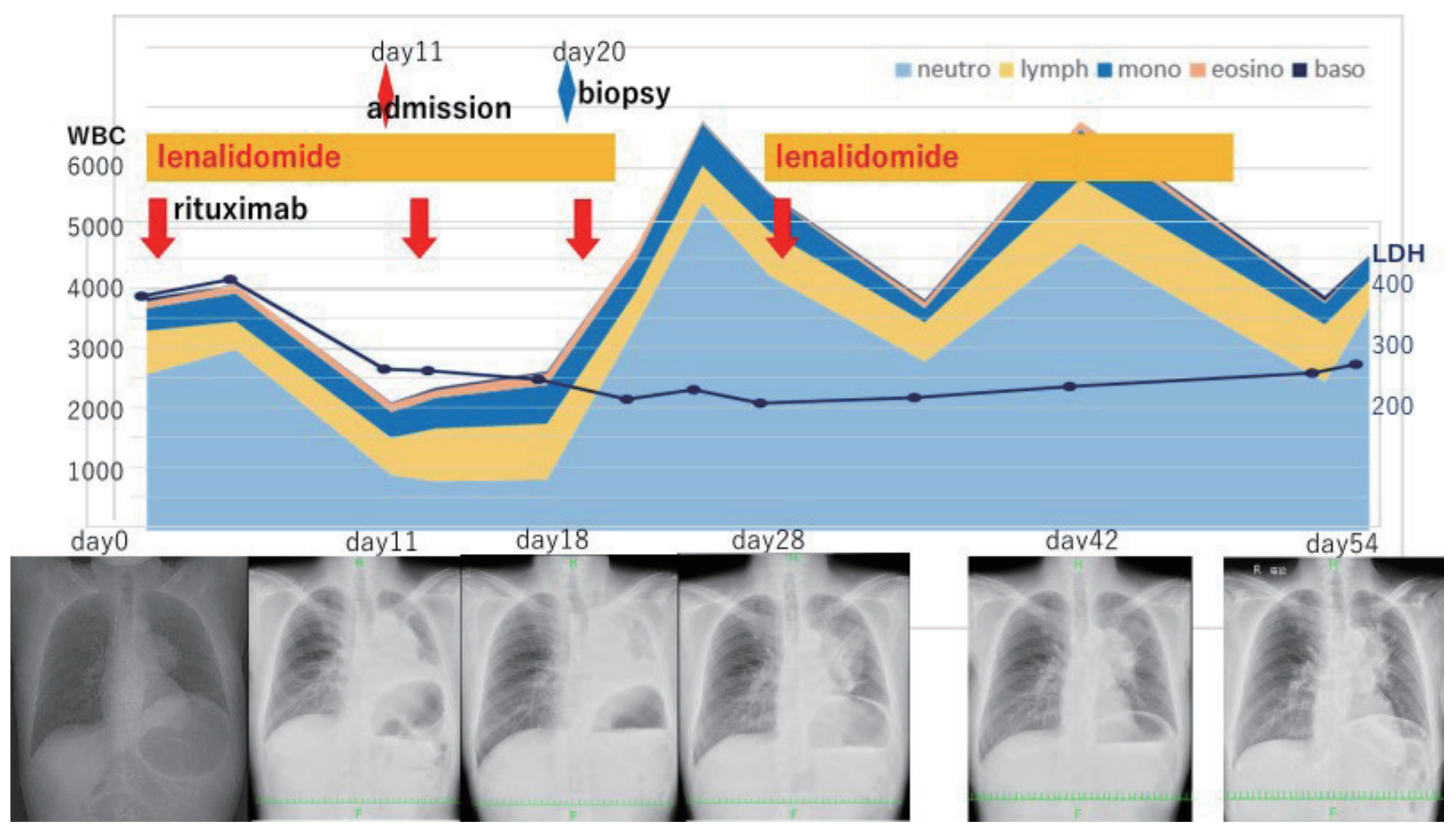

Fig. 3. Clinical course of $\mathrm{R}^{2}$ therapy: Chest X-ray on days $11,18,28,42$ and 54 . Chest imaging on day 0 was by CT because we did not perform chest X-ray at the start of $\mathrm{R}^{2}$ therapy.

Wang et al. found that lenalidomide with rituximab is effective even for transformed large cell lymphoma originating from follicular lymphoma. ${ }^{9}$ In a randomized study for relapsed/refractory diffuse large B-cell lymphoma, patients treated using lenalidomide had a longer progression-free survival than those treated at the investigator's discretion (gemcitabine, rituximab, etoposide or oxaliplatin). ${ }^{10}$ Based on the resistance to chemotherapy in the present case, we expected complete remission via a unique mechanism of action from the combination of lenalidomide and rituximab rather than the intensity of salvage chemotherapy. Therefore, we selected $\mathrm{R}^{2}$ therapy even if transformation was possible.

Immunotherapy, such as IMiDs and ICIs, works differently from chemotherapy and takes more time to exhibit effects than cytotoxic drugs. Chanan-Khan et al. reported that TFR induced by immunomodulatory drugs, such as lenalidomide, develops in $>90 \%$ of patients during the first treatment cycle and the median time to onset is 6 days. ${ }^{11}$ Goy et al. also found that TFR generally developed during the first cycle of lenalidomide, with few events during later cycles. ${ }^{12}$ Our patient exhibited a typical clinical course regarding the time of TFR. Steroids are used for the management of severe cases of TFR and prophylaxis. Chong et al. reported that patients who received once weekly low-dose $(10 \mathrm{mg})$ dexamethasone had fewer dose interruptions for TFR during the first 8 weeks of lenalidomide. ${ }^{13}$ Our patient did not receive dexamethasone, but he was administered 100 $\mathrm{mg}$ of hydrocortisone once just before the start of rituximab on day 1, day 13 and day 20. Hydrocortisone acts for a short time, unlike dexamethasone. The present patient did not receive any steroids after hydrocortisone on day 1 , until he received hydrocortisone on day 13. If dexamethasone had been administered weekly in the first course, TFR may have been less severe.

It is difficult to differentiate pseudo-progression due to TFR from true progression only by imaging, which largely relies on the tumor size. ${ }^{5}$ The identification of TFR is important to avoid the premature discontinuation of effective therapy because the intensity of TFR is correlated with the probability of achieving a complete response. ${ }^{11}$ TFR should be paid attention to in the early stage of treatment using immunomodulatory drugs.

\section{CONFLICT OF INTEREST}

The authors declare that they have no conflicts of interest.

\section{REFERENCES}

1 Chiu H, Trisal P, Bjorklund C, et al. Combination lenalidomiderituximab immunotherapy activates anti-tumour immunity and induces tumour cell death by complementary mechanisms of action in follicular lymphoma. Br J Haematol. 2019; 185 : 240-253.

2 Chanan-Khan AA, Chitta K, Ersing N, et al. Biological effects and clinical significance of lenalidomide-induced tumour flare reaction in patients with chronic lymphocytic leukaemia: in vivo evidence of immune activation and antitumour response. 
Br J Haematol. 2011; 155 : 457-467.

3 Chanan-Khan A, Miller KC, Takeshita K, et al. Results of a phase 1 clinical trial of thalidomide in combination with fludarabine as initial therapy for patients with treatment-requiring chronic lymphocytic leukemia (CLL). Blood. 2005; 106 : 3348-3352.

4 Chanan-Khan A, Miller KC, Musial L, et al. Clinical efficacy of lenalidomide in patients with relapsed or refractory chronic lymphocytic leukemia: results of a phase II study. J Clin Oncol. 2006; 24 : 5343-5349.

5 Taleb B A. Tumour flare reaction in cancer treatments: a comprehensive literature review. Anticancer Drugs. 2019; 30 : 953-958.

6 Wolchok JD, Hoos A, O'Day S, et al. Guidelines for the evaluation of immune therapy activity in solid tumors: immune-related response criteria. Clin Cancer Res. 2009; 15 : 7412-7420.

7 Andritsos LA, Johnson AJ, Lozanski G, et al. Higher doses of lenalidomide are associated with unacceptable toxicity including life-threatening tumor flare in patients with chronic lymphocytic leukemia. J Clin Oncol. 2008; 26 : 2519-2525.

8 Leonard JP, Trneny M, Izutsu K, et al. AUGMENT: a phase III study of lenalidomide plus rituximab versus placebo plus rituximab in relapsed or refractory indolent lymphoma. J Clin Oncol. 2019; 37 : 1188-1199.
9 Wang M, Fowler N, Wagner-Bartak N, et al. Oral lenalidomide with rituximab in relapsed or refractory diffuse large cell, follicular and transformed lymphoma: a phase II clinical trial. Leukemia. 2013; 27 : 1902-1909.

10 Czuczman MS, Trněný M, Davies A, et al. A phase 2/3 multicenter, randomized, open-label study to compare the efficacy and safety of lenalidomide versus investigator's choice in patients with relapsed or refractory diffuse large B-cell lymphoma. Clin Cancer Res. 2017; 23 : 4127-4137.

11 Chanan-Khan A, Miller KC, Lawrence D, et al. Tumor flare reaction associated with lenalidomide treatment in patients with chronic lymphocytic leukemia predicts clinical response. Cancer. 2011; $117: 2127-2135$.

12 Goy A, Sinha R, Williams ME, et al. Single-agent lenalidomide in patients with mantle-cell lymphoma who relapsed or progressed after or were refractory to bortezomib: phase II MCL001 (EMERGE) study. J Clin Oncol. 2013; 31 : 3688-3695.

13 Chong EA, Ahmadi T, Aqui NA, et al. Combination of lenalidomide and rituximab overcomes rituximab resistance in patients with indolent B-cell and mantle cell lymphomas. Clin Cancer Res. 2015; 21 : 1835-1842. 